\title{
General properties of cosmological models with an Isotropic Singularity
}

\author{
Geoffery Ericksson ${ }^{\ddagger} \quad$ Susan M. Scott ${ }^{\S}$
}

\begin{abstract}
Much of the published work regarding the Isotropic Singularity is performed under the assumption that the matter source for the cosmological model is a barotropic perfect fluid, or even a perfect fluid with a $\gamma$-law equation of state. There are, however, some general properties of cosmological models which admit an Isotropic Singularity, irrespective of the matter source. In particular, we show that the Isotropic Singularity is a point-like singularity and that vacuum space-times cannot admit an Isotropic Singularity. The relationships between the Isotropic Singularity, and the energy conditions, and the Hubble parameter is explored. A review of work by the authors, regarding the Isotropic Singularity, is presented.
\end{abstract}

\section{Introduction}

The concept of an Isotropic Singularity (IS) was introduced to the field of mathematical cosmology by Goode and Wainwright in 1985 [1]. Since that time much effort has been directed at investigating the physical consequences of the definition. This paper is devoted to an examination of some of the general properties of cosmological models which admit an IS. Previously published work by the authors, in this area, is reviewed in Section 4 Prior to that, for reference, the definition of an IS is given in Section 2 together with the associated definition of a cosmic time function. A proof follows which details a freedom in the choice of the conformal factor associated with an IS. For cosmologies comprising a fluid flow, the definitions of a fluid

\footnotetext{
${ }^{\ddagger}$ Advanced Computational Modelling Centre, The University of Queensland, Brisbane, QLD 4072. E-mail: gbe@acmc.uq.edu.au

${ }^{\S}$ Department of Physics, Faculty of Science, The Australian National University, Canberra, ACT 0200, Australia. E-mail: Susan. Scott@anu.edu.au
} 
congruence which is regular at an IS and a fluid congruence which is orthogonal to an IS are given in Section 3. When a cosmological model admits an IS at which the fluid flow is regular, it is shown that a conformal factor can always be chosen such that the expansion scalar associated with the unphysical fluid flow becomes zero at a given point in ${ }^{*} \mathcal{M}$ (with $T \geq 0$ ).

In Section 5 the issue of whether or not vacuum cosmologies can admit an IS is investigated. The IS is proven to be a point-like singularity, as one would expect, in Section [6. In Section 7 the Hubble parameter is shown to become infinite at the initial singularity of any cosmological model with an IS. The relationship between the energy conditions and the IS for perfect fluid cosmological models is examined in detail in Section 8 ,

\section{Definition of an Isotropic Singular- ity}

Following the introduction of the IS concept by Goode and Wainwright [1], Scott [4, 5, 3] amended their definition to remove some inherent redundancies. It is this amended definition which is given in Definition 2.1 and which will be used throughout this paper.

Definition 2.1 (Isotropic singularity) A space-time $(\mathcal{M}, \boldsymbol{g})$ is said to admit an isotropic singularity if there exists a space-time $\left({ }^{*} \mathcal{M},{ }^{*} \boldsymbol{g}\right)$, a smooth cosmic time function $T$ defined on ${ }^{*} \mathcal{M}$, and a conformal factor $\Omega(T)$ which satisfy

1. $\mathcal{M}$ is the open submanifold $T>0$,

2. $\boldsymbol{g}=\Omega^{2}(T)^{*} \boldsymbol{g}$ on $\mathcal{M}$, with ${ }^{*} \boldsymbol{g}$ regular (at least $C^{3}$ and non-degenerate) on an open neighbourhood of $T=0$,

3. $\Omega(0)=0$ and $\exists b>0$ such that $\Omega \in C^{0}[0, b] \cap C^{3}(0, b]$ and $\Omega(0, b]>0$,

4. $\lambda \equiv \lim _{T \rightarrow 0^{+}} L(T)$ exists, $\lambda \neq 1$, where $L \equiv \frac{\Omega^{\prime \prime}}{\Omega}\left(\frac{\Omega}{\Omega^{\prime}}\right)^{2}$ and a prime denotes differentiation with respect to $T$.

The definition of an isotropic singularity is described pictorially in Figure 1. The definition of a cosmic time function [3] 2, p198] which is used in conjunction with Definition [2.1, is the one given in Definition 2.2

Definition 2.2 (Cosmic time function) A function $T$ on ${ }^{*} \mathcal{M}$ is said to be a cosmic time function if ${ }^{*} \nabla T$ is everywhere timelike on ${ }^{*} \mathcal{M}$ with respect to ${ }^{*} \boldsymbol{g}$. 
(M,g)

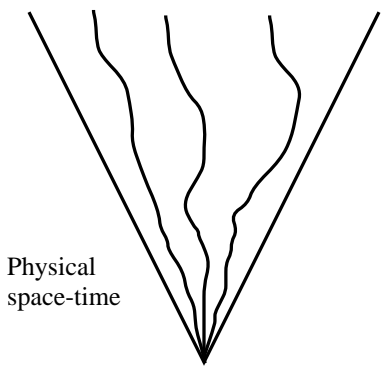

$(* \mathrm{M}, * \mathrm{~g})$

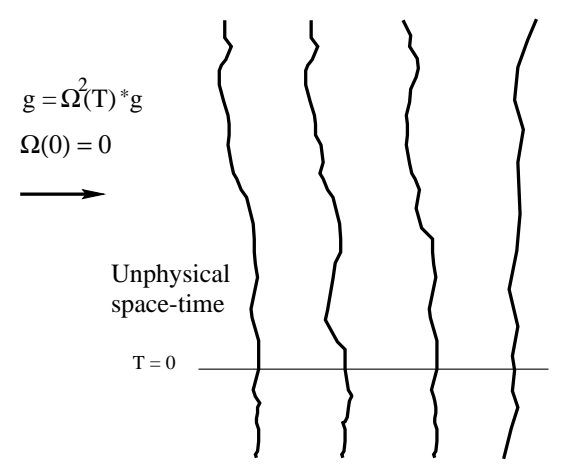

Figure 1: Pictorial interpretation of the isotropic singularity.

It should be noted that a cosmic time function defines a family of spacelike hypersurfaces $\{T=$ constant $\}$ in ${ }^{*} \mathcal{M}$ and hence in $\mathcal{M}$. A cosmic time function also increases along every future-directed nonspacelike curve.

Since $(\mathcal{M}, \mathbf{g})$ is the space-time in hand-in our case, typically, a cosmological solution of the Einstein field equations (EFE) - it is usual to call it the physical space-time. The conformally related space-time, $\left({ }^{*} \mathcal{M},{ }^{*} \mathrm{~g}\right)$, is then called the unphysical space-time.

Although the definition of an IS, and the arrow in Figure 1, lead one to think of the unphysical space-time being "created" from the physical space-time, it is actually useful to consider the situation in the reverse fashion. In this way, the singularity in the physical space-time should be regarded as arising due to the vanishing of the conformal factor, $\Omega(T)$, at the regular hypersurface $T=0$ in ${ }^{*} \mathcal{M}$.

Two noteworthy features of the definition of an IS are that it is coordinate-independent, as well as being independent of the EFE, and hence of the source of the gravitational field.

The conformal factor in the definition of an IS is not unique, as will be proven in the following Proposition. 
Proposition 2.1 The conformal factor associated with a particular IS can be multiplied by a factor of $e^{k(T)}$,

$$
\text { i.e., } \tilde{\Omega}(T)=e^{k(T)} \Omega(T),
$$

where $k(T)$ is a $C^{3}$ function of $T$ on $\mathbb{R}$, and still satisfy the conditions of the definition of an IS.

\section{Proof:}

An outline of this proof was given by Goode and Wainwright [1]. If the space-time $(\mathcal{M}, \mathbf{g})$ admits an IS, then there exists a conformally related space-time $\left({ }^{*} \mathcal{M},{ }^{*} \mathbf{g}\right)$ which satisfies the conditions of Definition 2.1. We now create a second unphysical space-time $\left({ }^{*} \mathcal{M},{ }^{*} \tilde{\mathrm{g}}\right)$ from the given unphysical space-time $\left({ }^{*} \mathcal{M},{ }^{*} \mathrm{~g}\right)$ by using the same manifold ${ }^{*} \mathcal{M}$ and a metric ${ }^{*} \tilde{\mathrm{g}}$ which is conformally related to ${ }^{*} \mathrm{~g}$ by the conformal factor $e^{k(T)}$, where $k(T)$ is a $C^{3}$ function of $T$ on $\mathbb{R}$, i.e., ${ }^{*} \mathbf{g}=e^{2 k(T) *} \tilde{\mathbf{g}}$. Note that the cosmic time function $T$ on ${ }^{*} \mathcal{M}$ is the same for both $\left({ }^{*} \mathcal{M},{ }^{*} \mathbf{g}\right)$ and $\left({ }^{*} \mathcal{M},{ }^{*} \tilde{g}\right)$. The metric $\mathbf{g}$ of the physical space-time $(\mathcal{M}, \mathbf{g})$ is related on $\mathcal{M}$ to the metric ${ }^{*} \mathrm{~g}$ of the second unphysical space-time $\left({ }^{*} \mathcal{M},{ }^{*} \tilde{\mathbf{g}}\right)$ by the conformal factor $\tilde{\Omega}=e^{k(T)} \Omega$, i.e., $\mathbf{g}=\tilde{\Omega}^{2}(T)^{*} \tilde{\mathbf{g}}$. Figure 2 shows the relationship between the physical space-time and the two unphysical space-times.

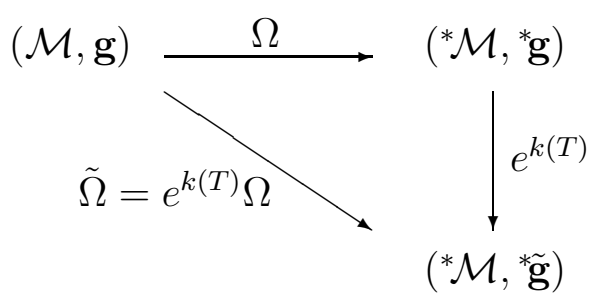

Figure 2: Relationship between the physical space-time and the two unphysical space-times.

Entities that exist in the second of the unphysical space-times, $\left({ }^{*} \mathcal{M},{ }^{*} \tilde{\mathrm{g}}\right)$, will be denoted by a star and an over-tilde, e.g., ${ }^{*} \tilde{A}$.

We now check each of the conditions outlined in Definition 2.1

1. $\mathcal{M}$ is still the open submanifold $T>0$ of ${ }^{*} \mathcal{M}$.

2. $\mathbf{g}=\tilde{\Omega}^{2}(T)^{*} \tilde{\mathbf{g}}$ on $\mathcal{M}$, where ${ }^{*} \tilde{\mathbf{g}}=e^{-2 k(T) *} \mathbf{g}$. Since ${ }^{*} \mathbf{g}$ is at least $C^{3}$ and non-degenerate on an open neighbourhood of $T=0$, and $e^{-2 k(T)}$ is a $C^{3}$ positive function of $T$ on $\mathbb{R}$, it follows that ${ }^{*} \mathbf{g}$ is at least $C^{3}$ and non-degenerate on an open neighbourhood of $T=0$. 
3. The relationship between the conformal factor $\Omega$ and the conformal factor $\tilde{\Omega}$ is given by $\tilde{\Omega}=e^{k(T)} \Omega$. We know that $\Omega(0)=0$ and $\exists b>0$ such that $\Omega \in C^{0}[0, b] \cap C^{3}(0, b]$ and $\Omega(0, b]>0$. Clearly $\tilde{\Omega}(0)=0$, and since $e^{k(T)}$ is a $C^{3}$ positive function of $T$ on $\mathbb{R}, \tilde{\Omega} \in C^{0}[0, b] \cap C^{3}(0, b]$ and $\tilde{\Omega}(0, b]>0$.

4.

$$
\begin{aligned}
\tilde{L}(T) & =\frac{\tilde{\Omega}^{\prime \prime} \tilde{\Omega}}{\left(\tilde{\Omega}^{\prime}\right)^{2}} \\
& =\frac{\Omega^{\prime \prime} \Omega+2 \Omega^{\prime} \Omega k^{\prime}+\Omega^{2} k^{\prime \prime}+\Omega^{2}\left(k^{\prime}\right)^{2}}{\left(\Omega^{\prime}+\Omega k^{\prime}\right)^{2}} \\
& =\frac{\frac{\Omega^{\prime \prime} \Omega}{\left(\Omega^{\prime}\right)^{2}}+2 k^{\prime} \frac{\Omega}{\Omega^{\prime}}+k^{\prime \prime}\left(\frac{\Omega}{\Omega^{\prime}}\right)^{2}+\left(k^{\prime}\right)^{2}\left(\frac{\Omega}{\Omega^{\prime}}\right)^{2}}{\left[1+\frac{\Omega}{\Omega^{\prime}} k^{\prime}\right]^{2}}
\end{aligned}
$$

It was shown by Scott [3] that $\lim _{T \rightarrow 0^{+}} \frac{\Omega}{\Omega^{\prime}}=0$. Now $k(T)$ is a $C^{3}$ function of $T$ on $\mathbb{R}$, and so $k^{\prime} \frac{\Omega}{\Omega^{\prime}} \rightarrow 0$ and $k^{\prime \prime}\left(\frac{\Omega}{\Omega^{\prime}}\right)^{2} \rightarrow 0$, as $T \rightarrow 0^{+}$. It follows that

$$
\tilde{L}(T)=\frac{\Omega^{\prime \prime} \Omega}{\left(\Omega^{\prime}\right)^{2}}[1+o(1)] \quad \text { as } T \rightarrow 0^{+},
$$

and therefore

$$
\lim _{T \rightarrow 0^{+}} \tilde{L}(T)=\lim _{T \rightarrow 0^{+}} L(T)=\lambda \quad(\neq 1) .
$$

The conditions for $\left({ }^{*} \mathcal{M},{ }^{*} \tilde{\mathrm{g}}\right)$ to be an unphysical space-time conformally related to $(\mathcal{M}, \mathbf{g})$ have now been satisfied.

\section{Fluid flow}

The definition of an Isotropic Singularity, as it stands, allows cosmological models to admit an "isotropic" singularity whose singularities are, in no sense, actually isotropic, or quasi-isotropic, or Friedmannlike [10, 1]. For example, the exact viscous fluid FRW cosmology of Coley and Tupper [11] can be shown to have an IS [1], and yet the shear and acceleration of the fluid flow are not dominated by its expansion as the singularity is approached, as one would expect with a Friedmann-like singularity. The fluid congruence is not regular at the IS in the Coley-Tupper models, however, which motivated Goode and Wainwright [1] to include the following additional definition relating to the fluid flow. 
Definition 3.1 (Fluid congruence) With any unit timelike congruence $\boldsymbol{u}$ in $\mathcal{M}$ we can associate a unit timelike congruence ${ }^{*} \boldsymbol{u}$ in ${ }^{*} \mathcal{M}$ such that

$$
{ }^{*} \boldsymbol{u}=\Omega \boldsymbol{u} \quad \text { in } \mathcal{M} .
$$

(a) If we can choose ${ }^{*} \boldsymbol{u}$ to be regular (at least $C^{3}$ ) on an open neighbourhood of $T=0$ in ${ }^{*} \mathcal{M}$, we say that $\boldsymbol{u}$ is regular at the isotropic singularity.

(b) If, in addition, ${ }^{*} \boldsymbol{u}$ is orthogonal to $T=0$, we say that $\boldsymbol{u}$ is orthogonal to the isotropic singularity.

It is the requirement that the fluid flow be regular at an IS (condition (a) of Definition 3.1), which ensures that the appropriate kinematic quantities behave as one would expect as an "isotropic" singularity is approached [1. In the remainder of this paper, unless stated otherwise, it will be assumed that any space-time which admits an IS has a fluid flow which is regular at the IS.

It should be noted that, in relation to Proposition 2.1 if the fluid congruence $\mathbf{u}$ is regular at an IS, then ${ }^{*} \mathbf{u}=\Omega \mathbf{u}$ (on $\mathcal{M}$ ) can be chosen to be at least $C^{3}$ on an open neighbourhood of $T=0$ in ${ }^{*} \mathcal{M}$. Since $k(T)$ is a $C^{3}$ function of $T$ on $\mathbb{R}$, then ${ }^{*} \tilde{\mathbf{u}}=e^{k(T) *} \mathbf{u}$ is also at least $C^{3}$ on an open neighbourhood of $T=0$ in ${ }^{*} \mathcal{M}$. It follows that $\mathbf{u}$ is also regular at the IS associated with the second unphysical space-time $\left({ }^{*} \mathcal{M},{ }^{*} \tilde{\mathrm{g}}\right)$.

In the following proposition we demonstrate how the freedom in the choice of conformal factor associated with an IS (see Proposition 2.11) can be used to ensure that the unphysical expansion scalar ${ }^{*} \theta$ is zero when evaluated at a given point $p$ in ${ }^{*} \mathcal{M}$ with $T \geq 0$. Goode and Wainwright 1 have previously examined the case of an irrotational barotropic perfect fluid model which admits an IS at which the fluid flow is regular, and the fluid flow is orthogonal to the family of spacelike hypersurfaces defined by $T=$ constant, where $T$ is the cosmic time function, and shown that the unphysical expansion scalar ${ }^{*} \theta$ is constant (with zero as one possible choice) on the $T=0$ hypersurface. Proposition 3.1 shows that a conformal factor can be chosen which sets ${ }^{*} \theta=0$ everywhere on this hypersurface.

Proposition 3.1 Suppose that the space-time $(\mathcal{M}, \boldsymbol{g})$ admits an IS at which the fluid flow $\boldsymbol{u}$ is regular. Let $p$ be a point (with $T \geq 0$ ) in the conformally related unphysical space-time $\left({ }^{*} \mathcal{M},{ }^{*} \boldsymbol{g}\right)$. Then by the suitable choice of a new conformal factor $\tilde{\Omega}$ (see Proposition [2.1) we can ensure that

$$
\left.{ }^{*} \tilde{\theta}\right|_{p}=0
$$




\section{Proof:}

For this proof we will use the conformal structure detailed in the proof of Proposition 2.1.

The expansion scalars $\theta$ and ${ }^{*} \theta$ for the two conformally related space-times $(\mathcal{M}, \mathbf{g})$ and $\left({ }^{*} \mathcal{M},{ }^{*} \mathrm{~g}\right)$ are related by

$$
{ }^{*} \theta=\Omega \theta-3 \frac{\Omega^{\prime}}{\Omega} T_{, a}^{*} u^{a} .
$$

The expansion scalar ${ }^{*} \tilde{\theta}$ for the second unphysical space-time $\left({ }^{*} \mathcal{M},{ }^{*} \tilde{\mathbf{g}}\right)$ is therefore related to the expansion scalar ${ }^{*} \theta$ for the first unphysical space-time $\left({ }^{*} \mathcal{M},{ }^{*} \mathrm{~g}\right)$ by

$$
\begin{aligned}
* \tilde{\theta} & =e^{k(T) *} \theta-3 k^{\prime} T_{, a}{ }^{*} \tilde{u}^{a} \\
& =e^{k(T)}\left[{ }^{*} \theta-3 k^{\prime} T_{, a}{ }^{*} u^{a}\right],
\end{aligned}
$$

where this relationship is valid on $\mathcal{M}$ (i.e., on ${ }^{*} \mathcal{M}$ for $T>0$ ), as well as on an open neighbourhood of $T=0$ in ${ }^{*} \mathcal{M}$. Thus, for $p \in{ }^{*} \mathcal{M}$ (with $T \geq 0$ ), we can arrange to have $\left.{ }^{*} \tilde{\theta}\right|_{p}=0$ by choosing the function $k(T)$ such that

$$
\left.k^{\prime}\right|_{T(p)}=\frac{1}{3} \frac{\left.{ }^{*} \theta\right|_{p}}{\left.\left(T_{, a}^{*} u^{a}\right)\right|_{p}},
$$

noting that $T_{, a}^{*} u^{a} \neq 0$.

\section{Previous Results}

It is interesting to review the main physical results which have been achieved using the amended version of the Goode and Wainwright definition of an IS given by Scott. It is fair to say that, until recently, the only major issue tackled with great success in this field was the vorticity problem. Goode [6] showed that if a perfect fluid solution of the EFE with an exact gamma-law equation of state $(1<\gamma<2)$ admits an IS at which the fluid flow is regular, then the fluid flow must be irrotational. Scott [3, 4, 5] has extended this result to include all barotropic perfect fluids with $-1<\lambda<1$. This result is known as the General Vorticity Result (GVR).

As well as establishing the GVR, Scott [3] examines in detail, and amends, the definition of an IS, and also determines the form of the conformal factor in terms of the cosmic time function. Amongst other results, Scott provides a rigorous proof that if a perfect fluid (not necessarily barotropic) solution of the EFE admits an IS $(\lambda \neq-\infty$ or $\frac{1}{2}$ ) at which the fluid flow is regular, then there exists a limiting gamma-law equation of state as the singularity is approached. The 
limiting behaviour, as the singularity is approached, of an acceleration potential for the fluid flow is also established for barotropic perfect fluids in this category.

Various examples of cosmological models which admit an isotropic singularity at which the fluid flow is regular are presented in the literature. A discussion of these models forms a review paper [7], which also includes a classification of the models according to the limiting behaviour, as the IS is approached, of their various kinematical properties and tensorial quantities.

Recently the authors have established the Zero Acceleration Result [8] (ZAR) for solutions of the EFE with barotropic perfect fluid source. This result says that if the fluid congruence of such a solution is shearfree and regular at an IS (with $-1<\lambda<1$ ), then the fluid flow is necessarily geodesic.

\section{Vacuum cosmologies}

If a solution of the Einstein field equations admits an IS, then it cannot be a vacuum solution*. This is easily seen by contrasting the fact that, globally, a vacuum solution satisfies

$$
R_{a}{ }^{b} R_{b}^{a}=0
$$

with the result, by Goode and Wainwright [1], that as an IS at $\mathrm{T}=0$ is approached,

$$
\lim _{T \rightarrow 0^{+}} R_{a}^{b} R_{b}^{a}=\infty .
$$

In light of this result we henceforth confine our attention to fluid filled space-times.

\section{Singularity type}

In order to gain some insight into the behaviour of the fluid around the singularity in a cosmological model we take a fluid element, which at some finite time is assumed to be spherical, and examine the asymptotic behaviour of this fluid element as we approach the initial singularity back along the fluid flow lines ${ }^{\dagger}$. To do this we look at length

\footnotetext{
${ }^{*}$ This result agrees with the analogous result, by Lifshitz and Khalatnikov [9, p203], for the quasi-isotropic singularity.

${ }^{\dagger}$ This approach was pioneered by Thorne [12, but this section is based on MacCallum [13. p131].
} 
scales $l_{\alpha}$ in the eigendirections ${ }^{\ddagger}$ of the expansion tensor. The length scales $l_{\alpha}(\alpha=1,2,3)$ are defined, up to a multiplicative constant, by

$$
\frac{i_{\alpha}}{l_{\alpha}} \equiv \theta_{\alpha}^{\alpha}, \quad \alpha \text { not summed }
$$

where $\theta^{\alpha}{ }_{\alpha}$ are the components of the expansion tensor in its eigenframe. An overall length scale (also called the scale factor) is defined by

$$
l=\left(l_{1} l_{2} l_{3}\right)^{\frac{1}{3}} \quad \text { or equivalently by } \quad \frac{i}{l}=\frac{\theta}{3} .
$$

A singularity is defined to be

- a point-like singularity if all three $l_{\alpha}$ approach zero,

- a barrel singularity if two of the $l_{\alpha}$ approach zero and the other approaches some finite number,

- a cigar singularity if two of the $l_{\alpha}$ approach zero and the other approaches infinity,

- a pancake singularity if one of the $l_{\alpha}$ approaches zero and the other two approach some finite number.

It should be noted that it is possible for a singularity to be none of the above singularity types. For example, the mixmaster cosmological models have the $l_{\alpha}$ oscillating as the singularity is approached.

From the above choices of singularity type, it would be natural to expect that the IS is a point-like singularity. Indeed, this is alluded to by Tod in a review paper [14] and proven for the case of a geodesic flow by Nolan [15.

Before we proceed to prove, in general, that the IS actually is a point-like singularity, we must first establish the following result.

Proposition 6.1 Suppose that the space-time $(\mathcal{M}, \boldsymbol{g})$ with a $C^{1}$ unit timelike congruence $\boldsymbol{u}$ admits an IS. We denote the corresponding $C^{1}$ unit timelike congruence in the unphysical space-time $\left({ }^{*} \mathcal{M},{ }^{*} \boldsymbol{g}\right)$ by ${ }^{*} \boldsymbol{u}=$ $\Omega \boldsymbol{u}$. For $p \in \mathcal{M}$, a vector, $\boldsymbol{v} \in T_{p}(\mathcal{M})$, where $\boldsymbol{v}$ is orthogonal to $\left.\boldsymbol{u}\right|_{p}$, is an eigenvector (with eigenvalue $\kappa$ ) of the expansion tensor, $\theta^{a}{ }_{b}$, of $\boldsymbol{u}$ if and only if it is an eigenvector (with eigenvalue ${ }^{*} \kappa$ ) of the expansion tensor, ${ }^{*} \theta^{a}{ }_{b}$, of ${ }^{*} \boldsymbol{u}$.

\section{Proof:}

We note firstly that since $\mathbf{u}$ is orthogonal to $\theta^{a}{ }_{b}$ and ${ }^{*} \theta^{a}{ }_{b}$, then $\mathbf{u}$ is an

\footnotetext{
${ }^{\ddagger}$ Note that the eigenvectors of the expansion tensor are in the same directions as the eigenvectors of the shear tensor.
} 
eigenvector of both $\theta^{a}{ }_{b}$ and ${ }^{*} \theta^{a}{ }_{b}$ with zero eigenvalues. That is, the eigenvalues $\kappa$ and ${ }^{*} \kappa$ are both zero for the eigenvector $\mathbf{u}$.

Now for $p \in \mathcal{M}$, coordinates $\left(x^{a}\right)$ about $p$ can be chosen so that $\left.\frac{\partial}{\partial x^{0}}\right|_{p}=\left.\mathbf{u}\right|_{p}$ and $\left.\mathbf{g}\left(\frac{\partial}{\partial x^{0}}, \frac{\partial}{\partial x^{\alpha}}\right)\right|_{p}=0(\alpha=1,2,3)$. It follows from this that $\left.{ }^{*} \mathbf{g}\left(\frac{\partial}{\partial x^{0}}, \frac{\partial}{\partial x^{\alpha}}\right)\right|_{p}=\left.{ }^{*} \mathbf{g}\left({ }^{*} \mathbf{u}, \frac{\partial}{\partial x^{\alpha}}\right)\right|_{p}=0$.

It is readily seen from the form of $\theta^{a}{ }_{b}$ and ${ }^{*} \theta^{a}{ }_{b}$ in these coordinates, that any eigenvectors of $\theta^{a}{ }_{b}$ at $p$, with non-zero eigenvalues, are orthogonal to $\mathbf{u}$, and any eigenvectors of ${ }^{*} \theta^{a}{ }_{b}$ at $p$, with non-zero eigenvalues, are orthogonal to ${ }^{*} \mathbf{u}$.

Now consider the vector $\beta \mathbf{u}+\mathbf{w} \in T_{p} \mathcal{M}$, where $\beta \neq 0$ and $\mathbf{w} \in$ $T_{p} \mathcal{M}$ is orthogonal to $\mathbf{u}$. If $\beta \mathbf{u}+\mathbf{w}$ is an eigenvector of $\theta^{a}{ }_{b}$ at $p$ with zero eigenvalue, then

$$
\begin{aligned}
\theta^{a}{ }_{b}\left(\beta u^{b}+w^{b}\right) & =0 \\
\theta_{b}^{a} w^{b} & =0
\end{aligned}
$$

That is, $\mathbf{w}$ is also an eigenvector of $\theta^{a}{ }_{b}$ at $p$ with zero eigenvalue.

We recall that the conformal relationship between the expansion tensor, $\theta^{a}{ }_{b}$, of $\mathbf{u}$ and the expansion tensor, ${ }^{*} \theta^{a}{ }_{b}$, of ${ }^{*} \mathbf{u}$ is given by

$$
\Omega \theta_{b}^{a}={ }^{*} \theta_{b}^{a}+{ }^{*} h^{a}{ }_{b} \Omega^{-1} \Omega_{, d} u^{d} .
$$

Now for $p \in \mathcal{M}$, suppose that $\mathbf{v}$ is an eigenvector of $\theta^{a}{ }_{b}$ at $p$ which is orthogonal to $\mathbf{u}$ :

$$
\begin{array}{ll}
\text { i.e. } & \theta^{a}{ }_{b} v^{b}=\kappa v^{a} \\
\Longleftrightarrow \quad & \Omega \theta^{a}{ }_{b} v^{b}=\Omega \kappa v^{a} \quad(\Omega>0) \\
\Longleftrightarrow & { }^{*} \theta^{a}{ }_{b} v^{b}+{ }^{*} h^{a}{ }_{b} v^{b} \Omega^{-1} \Omega, d^{*} u^{d}=\Omega \kappa v^{a} \\
\Longleftrightarrow \quad{ }^{*} \theta^{a}{ }_{b} v^{b}=\left(\Omega \kappa-\Omega^{-1} \Omega_{, d}{ }^{*} u^{d}\right) v^{a} \quad\left(\text { since }{ }^{*} h^{a}{ }_{b} v^{b}=v^{a}\right) \\
\Longleftrightarrow \quad{ }^{*} \theta_{b}{ }_{b} v^{b}={ }^{*} \kappa v^{a} \quad \text { where }{ }^{*} \kappa=\Omega \kappa-\Omega^{-1} \Omega_{, d}{ }^{*} u^{d}
\end{array}
$$

i.e., $\mathbf{v}$ is an eigenvector of ${ }^{*} \theta^{a}{ }_{b}$ at $p$ which is orthogonal to ${ }^{*} \mathbf{u}$.

Theorem 6.1 If the space-time $(\mathcal{M}, \boldsymbol{g})$ admits an IS at which the fluid flow is regular, then $(\mathcal{M}, \boldsymbol{g})$ has a point-like singularity.

Proof:

We recall that the expansion tensor, $\theta^{a}{ }_{b}$, of $\mathbf{u}$ for the physical spacetime $(\mathcal{M}, \mathbf{g})$ is related to its unphysical equivalent, ${ }^{*} \theta^{a}{ }_{b}$, of ${ }^{*} \mathbf{u}$ for the unphysical space-time $\left({ }^{*} \mathcal{M},{ }^{*} \mathrm{~g}\right)$ by the equation

$$
\theta_{b}^{a}=\Omega^{-1 *} \theta_{b}^{a}+\Omega^{-1 *} h_{b}^{a}(\ln \Omega)_{, d}{ }^{*} u^{d} .
$$

Choose an arbitrary flow line in ${ }^{*} \mathcal{M}$ and label it $\gamma$. The point $p \in \gamma$ lies on the IS hypersurface at $T=0$. We now proceed to construct 
a local comoving coordinate system $\left(s, x^{\alpha}\right)$ in $\left({ }^{*} \mathcal{M},{ }^{*} \mathrm{~g}\right)$. Set $s=z T$ along $\gamma$, where $T$ is the cosmic time function defined on ${ }^{*} \mathcal{M}$, and $z \in \mathbb{R}^{+}$is a constant. The coordinate $s$ will be strictly monotonically increasing up $\gamma$. Now consider a small open tube $\mathcal{N}$ of flow lines about $\gamma$ in ${ }^{*} \mathcal{M}$ and the hypersurfaces, within $\mathcal{N}$, which are orthogonal to the flow lines. The coordinate $s$ will be chosen to be constant across these orthogonal hypersurfaces. Note that the $s=$ constant hypersurfaces are not necessarily the same as the $T=$ constant hypersurfaces. The constant $z$ is chosen so that $\left.\frac{\partial}{\partial s}\right|_{p}=\left.{ }^{*} \mathbf{u}\right|_{p}$. On the hypersurface $s=0$ we choose spatial coordinates $x^{\alpha}$ which are comoving, i.e., the spatial coordinates along any flow-line remain constant.

One further restriction is placed on the spatial coordinates. They are chosen so that, at the point $p$ on the hypersurface $T=0$, the coordinate tangent vectors $\frac{\partial}{\partial x^{\alpha}}$ coincide with the three linearly independent eigenvectors, ${ }_{\alpha} \mathbf{v}$, at $p$, of the expansion tensor ${ }^{*} \theta^{a}{ }_{b}$ of the unphysical fluid flow, ${ }^{*} \mathbf{u}$. The eigenvectors, ${ }_{\alpha} \mathbf{v}$, are orthogonal to $\left.{ }^{*} \mathbf{u}\right|_{p}$. The eigenvectors ${ }^{*} \mathbf{u},{ }_{\alpha} \mathbf{v}$ of ${ }^{*} \theta^{a}{ }_{b}$ at $p$ can be extended to form a $C^{2}$ eigenvector frame $\left({ }^{*} \mathbf{u},{ }_{\alpha} \mathbf{v}\right)$ on $\mathcal{N}$, where the eigenvectors ${ }_{\alpha} \mathbf{v}$ are always orthogonal to ${ }^{*} \mathbf{u}$ at any point of $\mathcal{N}$. For points in $\mathcal{N}$, other than $p$, one would expect that ${ }_{\alpha} \mathbf{V}$ will not, in general, coincide with $\frac{\partial}{\partial x^{\alpha}}$, although both ${ }_{\alpha} \mathbf{v}$ and $\frac{\partial}{\partial x^{\alpha}}$ are orthogonal to ${ }^{*} \mathbf{u}$. It is certainly true, however, that, along $\gamma$

$$
{ }^{*} u^{0}=1+o(1), \quad{ }^{*} u^{\beta}=0, \quad{ }_{\alpha} v^{0}=0, \quad{ }_{\alpha} v^{\beta}=\delta^{\beta}{ }_{\alpha}+o(1) \quad \text { as } s \rightarrow 0^{+} .
$$

The coordinate tangent vector frame is thus arbitrarily close to the expansion tensor ${ }^{*} \theta^{a}{ }_{b}$ eigenvector frame as we approach $p$ down the flow line $\gamma$. Since, by Proposition 6.1, the expansion tensor ${ }^{*} \theta_{b}{ }_{b}$ eigenvector frame is the same as the expansion tensor $\theta^{a}{ }_{b}$ eigenvector frame, it follows that the coordinate tangent vector frame is also arbitrarily close to the expansion tensor $\theta^{a}{ }_{b}$ eigenvector frame as we approach $p$ down the flow line $\gamma$. A leading subscript "e" will be placed on any tensor evaluated in the expansion tensor $\theta^{a}{ }_{b}$ eigenvector frame.

We will now consider the relationship between the components of the expansion tensor $\theta^{a}{ }_{b}$ in its eigenvector frame and its components in the coordinate tangent vector frame. For $\alpha=1,2,3$, Equation (25) can be written

$$
\Omega \theta^{\alpha}{ }_{\alpha}={ }^{*} \theta^{\alpha}{ }_{\alpha}+{ }^{*} h^{\alpha}{ }_{\alpha} \frac{\Omega^{\prime}}{\Omega}\left({ }^{*} \nabla T\right)_{d}{ }^{*} u^{d}, \quad \alpha \text { not summed. }
$$

The corresponding equation in the eigenvector frame is

$$
\left.\Omega_{e} \theta^{\alpha}{ }_{\alpha}={ }_{e}^{*} \theta^{\alpha}{ }_{\alpha}+{ }_{e}^{*} h^{\alpha}{ }_{\alpha} \frac{\Omega^{\prime}}{\Omega} e^{*} \nabla T\right) d e^{*} u^{d} .
$$


As we approach $p$ down the flow line $\gamma$ in ${ }^{*} \mathcal{M}$,

$$
\begin{aligned}
{ }_{e}^{*} \theta^{\alpha}{ }_{\alpha} & ={ }^{*} \theta^{\alpha}{ }_{\alpha}[1+o(1)], \\
{ }^{*} h^{\alpha}{ }_{\alpha} & ={ }^{*} h^{\alpha}{ }_{\alpha}[1+o(1)], \\
\left.e^{*} \nabla T\right)_{d} & \left.={ }^{*} \nabla T\right)_{d}[1+o(1)], \\
{ }^{*} u^{d} & ={ }^{*} u^{d}[1+o(1)] .
\end{aligned}
$$

It follows that

$$
\Omega_{e} \theta_{\alpha}^{\alpha}=\Omega \theta_{\alpha}^{\alpha}[1+o(1)] \quad \text { as } s \rightarrow 0^{+},
$$

and thus

$$
e^{\theta^{\alpha}}{ }_{\alpha}=\theta^{\alpha}{ }_{\alpha}[1+o(1)] \quad \text { as } s \rightarrow 0^{+} .
$$

In the physical expansion tensor, $\theta^{a}{ }_{b}$, eigenvector frame the length scales, $l_{\alpha}(\alpha=1,2,3)$, are defined by $\frac{i_{\alpha}}{l_{\alpha}}={ }_{e} \theta^{\alpha}{ }_{\alpha}$, where $\alpha$ is not summed. We therefore have the relationship

$$
\frac{i_{\alpha}}{l_{\alpha}}=\theta_{\alpha}^{\alpha}[1+o(1)] \quad \text { as } s \rightarrow 0^{+},
$$

which in conjunction with Equation (27) yields

$$
\Omega \frac{i_{\alpha}}{l_{\alpha}}=\left({ }^{*} \theta^{\alpha}{ }_{\alpha}+{ }^{*} h^{\alpha}{ }_{\alpha} \frac{\Omega^{\prime}}{\Omega}\left({ }^{*} \nabla T\right)_{d}{ }^{*} u^{d}\right)[1+o(1)] .
$$

Now as we approach $p$ down the flow line $\gamma$ in ${ }^{*} \mathcal{M},\left.{ }^{*} \theta^{\alpha}{ }_{\alpha}\right|_{p} \in \mathbb{R}$, $\lim _{s \rightarrow 0^{+}} \frac{\Omega^{\prime}}{\Omega}=+\infty($ see Scott $[3])$, and

$$
\lim _{s \rightarrow 0^{+}}{ }^{*} h_{\alpha}^{\alpha}\left({ }^{*} \nabla T\right)_{d}{ }^{*} u^{d}=\lim _{s \rightarrow 0^{+}}\left({ }^{*} \nabla T\right)_{d}{ }^{*} u^{d} \in \mathbb{R} \backslash\{0\} .
$$

It follows that

$$
\begin{array}{rlrl} 
& & \dot{l}_{\alpha} & =\frac{\Omega^{\prime}}{\Omega}\left({ }^{*} \nabla T\right)_{d}{ }^{*} u^{d}[1+o(1)] \quad \text { as } s \rightarrow 0^{+} \\
\Leftrightarrow & \quad \Omega \frac{\dot{l}_{\alpha}}{l_{\alpha}} & =\Omega \frac{\Omega^{\prime}}{\Omega}(\nabla T)_{d} u^{d}[1+o(1)] \\
\Leftrightarrow & \Omega\left(\ln l_{\alpha}\right)^{\cdot} & =\Omega(\ln \Omega)^{\cdot}[1+o(1)] \\
\Leftrightarrow & \left(\ln l_{\alpha}\right)^{\cdot} & =(\ln \Omega)^{*}[1+o(1)] .
\end{array}
$$

Thus $\lim _{s \rightarrow 0^{+}} \frac{\left(\ln l_{\alpha}\right)^{\circ}}{(\ln \Omega)^{*}}=1$.

Since $\ln \Omega \rightarrow-\infty$ as $s \rightarrow 0^{+}$, L'Hôpital's rule can be invoked to obtain

$$
\begin{array}{rlrl} 
& & \lim _{s \rightarrow 0^{+}} \frac{\ln l_{\alpha}}{\ln \Omega} & =1 \\
\text { i.e. } & \ln l_{\alpha} & =\ln \Omega[1+o(1)] \quad \text { as } s \rightarrow 0^{+} \\
\Rightarrow & l_{\alpha} & \rightarrow & 0 \text { as } s \rightarrow 0^{+} .
\end{array}
$$


Note that the length scales, $l_{\alpha}(\alpha=1,2,3)$, have the same asymptotic form, as one would expect with an IS. Since $\gamma$ was an arbitrary flow line, we conclude that all three length scales $l_{\alpha}$ approach zero as the initial singularity is approached back along any fluid flow line. Thus the space-time $(\mathcal{M}, \mathbf{g})$ has a point-like singularity.

This theorem shows that if a cosmological model admits an IS at which the fluid flow is regular, then a spherical fluid element which travels backwards in time to the initial singularity, will collapse, approximately isotropically, into a point.

\section{Hubble parameter}

The Hubble parameter is an important parameter in comparing observational data with cosmological theory. It is defined by MacCallum [13. p97] as

$$
H \equiv \frac{i}{l}=\frac{\theta}{3}
$$

where $l$ is the scale factor.

The behaviour of the expansion scalar, $\theta$, can be determined as follows. Equation (38), from the proof of Theorem 6.1 shows that for a cosmological model which admits an IS, at which the fluid flow is regular, the following relationship holds down a fluid flow line:

$$
\frac{i_{\alpha}}{l_{\alpha}}=\frac{1}{\Omega} \frac{\Omega^{\prime}}{\Omega}\left({ }^{*} \nabla T\right)_{d}{ }^{*} u^{d}[1+o(1)] \quad \text { as } T \rightarrow 0^{+} .
$$

Since $\lim _{T \rightarrow 0^{+}} \frac{\Omega^{\prime}}{\Omega^{2}}=+\infty$ and $\lim _{T \rightarrow 0^{+}}\left({ }^{*} \nabla T\right)_{d}{ }^{*} u^{d} \in \mathbb{R}^{+}$, it follows that

$$
\frac{i_{\alpha}}{l_{\alpha}} \rightarrow+\infty \quad \text { as } T \rightarrow 0^{+} .
$$

Now

$$
\begin{aligned}
\theta & =\frac{i_{1}}{l_{1}}+\frac{i_{2}}{l_{2}}+\frac{i_{3}}{l_{3}} \\
\Rightarrow \quad \theta & \rightarrow+\infty \text { as } T \rightarrow 0^{+} .
\end{aligned}
$$

This result was originally obtained by Goode and Wainwright 1 .

Since the expansion scalar becomes infinite as the singularity is approached along any fluid flow line, it follows that

$$
H \rightarrow+\infty \quad \text { as } T \rightarrow 0^{+}
$$




\section{$8 \quad$ Energy conditions}

A solution of the EFE should be "physically reasonable" in order to be a candidate as a model for a real physical system. Although the terminology "physically reasonable" is vague, the standard method for determining whether or not a model is physically reasonable is to test if the energy conditions are satisfied. For a perfect fluid the usual energy conditions are [2, pp88-96]:

Weak Energy Condition (WEC): $\quad \mu \geq 0, \quad \mu+p \geq 0$.

Dominant Energy Condition (DEC): $\quad \mu \geq 0, \quad-\mu \leq p \leq \mu$.

Strong Energy Condition (SEC): $\quad \mu+p \geq 0, \quad \mu+3 p \geq 0$.

Scott [3] has shown that if a space-time satisfies the EFE with perfect fluid source, and the unit timelike fluid congruence is regular at an IS, then there is a relationship between the energy density, $\mu$, and the pressure, $p$, of the fluid near the singularity. The following two propositions give this relationship precisely.

Proposition 8.1 (Limiting $\gamma$-law result) If the space-time $(\mathcal{M}, \boldsymbol{g})$ is a $C^{3}$ solution of the EFE with perfect fluid source, and the unit timelike fluid congruence, $\boldsymbol{u}$, is regular at an IS $\left(\lambda \neq-\infty\right.$ or $\left.\frac{1}{2}\right)$, then there exists a limiting $\gamma$-law equation of state $p=(\gamma-1) \mu$ as the singularity is approached, where $\gamma=\frac{2}{3}(2-\lambda)$.

Proposition $8.2(\lambda=-\infty)$ If the space-time $(\mathcal{M}, \boldsymbol{g})$ is a $C^{3}$ solution of the EFE with perfect fluid source, and the unit timelike fluid congruence, $\boldsymbol{u}$, is regular at an IS with $\lambda=-\infty$, then there exists a limiting equation of state $p=-\frac{2}{3} L \mu$ as the singularity is approached, where $L(T)=\frac{\Omega^{\prime \prime} \Omega}{\left(\Omega^{\prime}\right)^{2}}$, and $\lim _{T \rightarrow 0^{+}} L(T)=\lambda$.

Using these results, we have determined, in the following corollary, whether, for perfect fluid cosmologies which admit an IS at which the fluid flow is regular, the above energy conditions are satisfied near the singularity.

Corollary 8.1 If the space-time $(\mathcal{M}, \boldsymbol{g})$ is a $C^{3}$ solution of the EFE with perfect fluid source, and the unit timelike fluid congruence, $\boldsymbol{u}$, is regular at an Isotropic Singularity, then there exists an open neighbourhood $\mathcal{U}$ of the hypersurface $T=0$ in ${ }^{*} \mathcal{M}$ such that the weak and strong energy conditions are satisfied everywhere on $\mathcal{U} \cap \mathcal{M}$. Furthermore, if $-1<\lambda<1$, then the dominant energy condition also holds on $\mathcal{U} \cap \mathcal{M}$. 


\section{Proof:}

Scott [3] has shown that, for space-times which satisfy the conditions of this corollary, $\mu \rightarrow+\infty$ as $T \rightarrow 0^{+}$, and hence $\mu \geq 0$ near the singularity.

From the limiting $\gamma$-law result of Scott we know that when $\lambda \neq-\infty$ or $\frac{1}{2}, p \approx(\gamma-1) \mu$ as $T \rightarrow 0^{+}$, with $\frac{2}{3}<\gamma<\infty(\gamma \neq 1)$. We will prove this case first, then look at the remaining $\lambda=\frac{1}{2}$ and $\lambda=-\infty$ cases.

1. Assume $\lambda \neq-\infty$ or $\frac{1}{2}$.

An open neighbourhood $\mathcal{U}$ of the hypersurface $T=0$ in ${ }^{*} \mathcal{M}$ can be chosen such that $\mu>0$ and $p \approx(\gamma-1) \mu$ as $T \rightarrow 0^{+}$on $\mathcal{U} \cap \mathcal{M}$. There are three subcases to examine.

(a) Show $\mu+p \geq 0$ on $\mathcal{U} \cap \mathcal{M}$ :

$$
\begin{aligned}
\mu+p & =\mu+(\gamma-1) \mu[1+o(1)] \\
& =\gamma \mu[1+o(1)] .
\end{aligned}
$$

Now $\gamma>\frac{2}{3}$ and $\mu>0$ on $\mathcal{U} \cap \mathcal{M}$, with $\mu \rightarrow+\infty$ as $T \rightarrow 0^{+}$. Hence $\mu+p \geq 0$ on $\mathcal{U} \cap \mathcal{M}$ and the WEC is satisfied.

(b) Show $\mu+3 p \geq 0$ on $\mathcal{U} \cap \mathcal{M}$ :

Proceeding as in case (1a), we can show that

$$
\mu+3 p=(3 \gamma-2) \mu[1+o(1)],
$$

and since $\gamma>\frac{2}{3}, 3 \gamma-2>0$. Hence $\mu+3 p \geq 0$ on $\mathcal{U} \cap \mathcal{M}$ and the SEC is satisfied.

(c) Establish when $-\mu \leq p \leq \mu$ holds on $\mathcal{U} \cap \mathcal{M}$ :

We have already shown that $-\mu \leq p$ on $\mathcal{U} \cap \mathcal{M}$, and so we need to determine when it is also true that $p \leq \mu$.

Since $p=(\gamma-1) \mu[1+o(1)]$ as $T \rightarrow 0^{+}$on $\mathcal{U} \cap \mathcal{M}$,

$$
\begin{aligned}
& p \leq \mu \\
\Leftrightarrow \quad & (\gamma-1) \mu[1+o(1)] \leq \mu \\
\Leftrightarrow \quad & (\gamma-1)[1+o(1)] \leq 1 \\
& \quad(\text { since } \mu>0 \text { on } \mathcal{U} \cap \mathcal{M}) .
\end{aligned}
$$

When $\gamma-1<1$, an open neighbourhood $\mathcal{U}$ of $T=0$ in ${ }^{*} \mathcal{M}$ always exists such that this inequality holds on $\mathcal{U} \cap \mathcal{M}$. On the other hand, when $\gamma-1>1$, such a neighbourhood never exists, since the inequality is not true in the limit as $T \rightarrow 0^{+}$. For the special value $\gamma=2$, the inequality may or may not hold in the limit as $T \rightarrow 0^{+}$. We conclude that, for $\lambda \neq-\infty$ or $\frac{1}{2}$, the DEC holds on $\mathcal{U} \cap \mathcal{M}$ when $\frac{2}{3}<\gamma<2(\gamma \neq 1)$, equivalently, $-1<\lambda<1\left(\lambda \neq \frac{1}{2}\right)$. 
2. Assume $\lambda=\frac{1}{2}$.

This case includes the dust $(p=0)$ and asymptotic dust $(p=$ $o(1)$ as $\left.T \rightarrow 0^{+}\right)$models. It is readily seen from [3] that $p=$ $o(\mu)$ as $T \rightarrow 0^{+}$. Let $\mathcal{U}$ denote an open neighbourhood of the hypersurface $T=0$ in ${ }^{*} \mathcal{M}$ such that $\mu>0$ and $p=o(\mu)$ as $T \rightarrow 0^{+}$on $\mathcal{U} \cap \mathcal{M}$. Since $\mu \rightarrow+\infty$ as $T \rightarrow 0^{+}$, it is clear that $\mu+p \geq 0, \mu+3 p \geq 0$, and $p \leq \mu$ on $\mathcal{U} \cap \mathcal{M}$. Thus, for the case $\lambda=\frac{1}{2}$, the WEC, SEC and DEC are satisfied on $\mathcal{U} \cap \mathcal{M}$.

3. Assume $\lambda=-\infty$.

In this case the limiting equation of state is given by

$$
p \approx-\frac{2}{3} L \mu \quad \text { as } T \rightarrow 0^{+},
$$

where $L(T)=\frac{\Omega^{\prime \prime} \Omega}{\left(\Omega^{\prime}\right)^{2}}$, and $\lim _{T \rightarrow 0^{+}} L(T)=\lambda$. An open neighbourhood $\mathcal{U}$ of the hypersurface $T=0$ in ${ }^{*} \mathcal{M}$ can be chosen such that $\mu>0, L<0$, and $p \approx-\frac{2}{3} L \mu$ as $T \rightarrow 0^{+}$on $\mathcal{U} \cap \mathcal{M}$.

Again there are three subcases to examine.

(a) Show $\mu+p \geq 0$ on $\mathcal{U} \cap \mathcal{M}$ :

$$
\begin{aligned}
\mu+p & =\mu-\frac{2}{3} L \mu[1+o(1)] \\
& =-\frac{2}{3} L \mu[1+o(1)] .
\end{aligned}
$$

Now $\mu>0$ and $L<0$ on $\mathcal{U} \cap \mathcal{M}$, with $-\frac{2}{3} L \mu \rightarrow+\infty$ as $T \rightarrow 0^{+}$. Hence $\mu+p \geq 0$ on $\mathcal{U} \cap \mathcal{M}$ and the WEC is satisfied.

(b) Show $\mu+3 p \geq 0$ on $\mathcal{U} \cap \mathcal{M}$ :

Proceeding as in case (3a), we can show that

$$
\mu+3 p=-2 L \mu[1+o(1)]
$$

Now $\mu>0$ and $L<0$ on $\mathcal{U} \cap \mathcal{M}$, with $-2 L \mu \rightarrow+\infty$ as $T \rightarrow 0^{+}$. Hence $\mu+3 p \geq 0$ on $\mathcal{U} \cap \mathcal{M}$ and the SEC is satisfied.

(c) Establish whether $-\mu \leq p \leq \mu$ holds on $\mathcal{U} \cap \mathcal{M}$ :

We have already shown that $-\mu \leq p$ on $\mathcal{U} \cap \mathcal{M}$, and so we need to determine if it is also true that $p \leq \mu$.

Since $p=-\frac{2}{3} L \mu[1+o(1)]$ as $T \rightarrow 0^{+}$on $\mathcal{U} \cap \mathcal{M}$,

$$
\begin{aligned}
& p \leq \mu \\
\Leftrightarrow \quad & -\frac{2}{3} L \mu[1+o(1)] \leq \mu \\
\Leftrightarrow \quad & -\frac{2}{3} L[1+o(1)] \leq 1 \\
& \quad(\text { since } \mu>0 \text { on } \mathcal{U} \cap \mathcal{M}) \\
\Leftrightarrow & 1+o(1) \leq-\frac{3}{2} \frac{1}{L} .
\end{aligned}
$$


An open neighbourhood $\mathcal{U}$ of $T=0$ in ${ }^{*} \mathcal{M}$ does not exist such that this inequality holds on $\mathcal{U} \cap \mathcal{M}$, since $-\frac{3}{2} \frac{1}{L} \rightarrow 0^{+}$ as $T \rightarrow 0^{+}$. We conclude that the DEC does not hold when $\lambda=-\infty$, which is what one would expect given our results for the general case (11) when $\lambda \neq-\infty$ or $\frac{1}{2}$.

The dominant energy condition may hold when $\gamma=2$, but this must usually be examined on a case by case basis. We can, however, state that any perfect fluid space-time with the exact $\gamma$-law equation of state, $p=\mu$, which admits an IS at which the fluid flow is regular, will satisfy the DEC. For example, the stiff fluid FRW models, and the stiff fluid Mars95 models [16], admit an IS at which the fluid flow is regular [7, and references therein] and satisfy the DEC. On the other hand, perfect fluid models with an equation of state $p=\mu[1+N]$, where $N=o(1)$ as $T \rightarrow 0^{+}, N>0$, which admit an IS at which the fluid flow is regular, do not satisfy the DEC.

\section{Conclusion}

As in any field, there is a certain amount of "folk lore" in general relativity. In particular, with regard to initial singularities in cosmological models, it has always been assumed that the IS is a point-like singularity and that vacuum space-times were of no concern in matters regarding the IS, in that they could not possess an IS. In this paper we have explicitly proven these two fundamental results. In addition to this we have shown that, near the initial singularity, the weak and strong energy conditions are automatically satisfied by perfect fluid space-times which admit an IS, and that the dominant energy condition is also satisfied given a reasonable restriction on the relationship between the pressure and the energy density. We have provided a detailed proof of the result, formerly stated by Goode and Wainwright, that there is a freedom in the choice of conformal factor associated with a space-time which admits an IS. This freedom was exploited to prove that the expansion scalar associated with an unphysical fluid flow can always be set to zero at a given point in ${ }^{*} \mathcal{M}$ by a suitable choice of the conformal factor. Finally, it was shown that the Hubble parameter becomes infinite at an IS.

\section{References}

[1] S. W. Goode and J. Wainwright "Isotropic singularities in cosmological models" Class. Quantum Grav. 2 (1985) pp99-115 
[2] S. W. Hawking and G. F. R. Ellis, The Large Scale Structure of Space-Time (Cambridge: Cambridge University Press, 1973).

[3] S. M. Scott to be published

[4] S. M. Scott "Isotropic singularities - the definition and vorticity" Proceedings of the 2nd Canadian Conference on General Relativity and Relativistic Astrophysics ed. A. Coley, C. Dyer, and B. Tupper (Singapore and New Jersey: World Scientific) (1988) pp373-6

[5] S. M. Scott "Conjectures related to the Weyl Curvature Hypothesis" Proceedings of the Fifth Marcel Grossmann Meeting on General Relativity (1988) ed. D. G. Blair and M. J. Buckingham (World Scientific) (1989) pp1033-6

[6] S. W. Goode "Vorticity and isotropic singularities" Gen. Rel. Grav. 19 (1987) pp1075-82

[7] S. M. Scott and G. Ericksson "Cosmological models with isotropic singularities" Proceedings of the International Seminar on Mathematical Cosmology, Potsdam March 30 - April 4, 1998 ed. M. Rainer, and H.-J. Schmidt (Singapore: World Scientific PC) (1998) pp123-32

[8] G. Ericksson and S. M. Scott "Isotropic singularities in shear-free perfect fluid cosmologies" Gen. Rel. Grav. 32 (2000) pp425-43

[9] E. M. Lifshitz and I. M. Khalatnikov "Investigations in relativistic cosmology" Adv. Phys. 12 (1963) pp185-249

[10] S. W. Goode Ph.D. thesis University of Waterloo (1983)

[11] A. A. Coley and B. O. J. Tupper "An exact viscous fluid FRW cosmology" Phys. Lett. 95A (1983) pp357-60

[12] K. P. Thorne "Primordial element formation, primordial magnetic fields, and the isotropy of the universe" Astrophys. J. 148 (1967) pp51-68

[13] M. MacCallum "Cosmological models from a geometric point of view" Cargese Lectures in Physics 6 ed. E. Schatzmann (New York: Gordon and Breach) (1973) pp61-174

[14] K. P. Tod "Isotropic singularities" Rend. Sem. Mat. Univ. Pol. Torino 50,1 (1992) pp69-92

[15] B. C. Nolan "A note on behaviour at an isotropic singularity" Class. Quantum Grav. 18 (2001) pp1127-36

[16] M. Mars "New non-separable diagonal cosmologies" Class. Quantum Grav. 12 (1995) pp2831-47 